\title{
Low-Dose Exposure of C57BL/6 Mice to Burkholderia pseudomallei Mimics Chronic Human Melioidosis
}

\author{
Laura Conejero, ${ }^{*}$ Natasha Patel, ${ }^{*}$ \\ Melanie de Reynal, ${ }^{*}$ Sara Oberdorf, ${ }^{*}$ \\ Joanne Prior, ${ }^{\dagger}$ Philip L. Felgner, ${ }^{\ddagger}$ \\ Richard W. Titball, ${ }^{\S}$ Francisco J. Salguero, " and \\ Gregory J. Bancroft* \\ From the Department of Immunology and Infection, * Faculty of \\ Infectious \& Tropical Diseases, London School of Hygiene $\&$ \\ Tropical Medicine, London, United Kingdom; the Defence Science \\ and Technology Laboratory, ${ }^{\dagger}$ Porton Down, Salisbury, United \\ Kingdom; the Department of Medicine, ${ }^{\neq}$University of California, \\ Irvine, Irvine, California; the College of Life and Environmental \\ Sciences, ${ }^{\circledR}$ University of Exeter, Exeter, United Kingdom; and \\ Veterinary Laboratories Agency-Weybridge," Addlestone, Surrey, \\ United Kingdom
}

Burkbolderia pseudomallei is the etiological agent of human melioidosis, a disease with a broad spectrum of clinical manifestations ranging from fatal septicemia to chronic localized infection or asymptomatic latent infection. Most clinical and immunological studies to date have focused on the acute disease process; however, little is known about pathology and immune response in chronic melioidosis. Here, we have developed a murine model of chronic disease by challenging C57BL/6 mice intranasally with a low dose of $B$. pseudomallei and monitoring them up to 100 days postinfection. Bacterial burdens were heterogeneous in different animals at all time points, consistent with the spectrum of clinical severity observed in humans. Proinflammatory cytokines such as gamma interferon (IFN- $\gamma$ ), interleukin-6 (IL-6), monocyte chemotactic protein-1 (MCP-1), and tumor necrosis factor- $\alpha$ (TNF- $\alpha$ ) were induced during chronic infection, and histopathological analysis showed features in common with human melioidosis. Interestingly, many of these features were similar to those induced by Mycobacterium tuberculosis in humans, such as development of a collagen cord that encapsulates the lesions, the presence of multinucleated giant cells, and granulomas with a caseous necrotic center, which may explain why chronic melioidosis is often misdiagnosed as tuberculosis. Our model now provides a relevant and practical tool to define the immunological features of chronic melioidosis and aid in the development of more effective treatment of this disease in humans. ( $A m \mathrm{~J}$ Patbol 2011, 179:270-280; DOI: 10.1016/j.ajpath.2011.03.031)

Burkholderia pseudomallei is a Gram-negative soil bacterium that is the causative agent of melioidosis. This pathogen is endemic in Southeast Asia and Northern Australia, but cases of melioidosis are now being reported in numerous other tropical areas, suggesting a more global distribution of the organism in the environment. ${ }^{1,2}$ The main routes of infection are believed to be via inhalation of aerosols during the rainy season or via cutaneous inoculation in people who have direct contact with wet soil. ${ }^{3}$ The consequences of infection with $B$. pseudomallei are remarkably diverse, ranging from an acute septicemia to a chronic localized disease or an asymptomatic latent infection. Acute infection is characterized by bacteremia, progression to septic shock with the induction of a massive but ineffective cytokine response, and a high mortality rate ${ }^{3-5}$ Chronic infection is generally less severe and often characterized by persistent, localized infection that is difficult to eradicate. ${ }^{3,6}$ Recurrent disease is also common, and it is mostly due to relapse rather than reinfection. ${ }^{7}$ The disease can also reactivate after decades of clinical latency, ${ }^{8}$ suggesting that the bacteria may enter a dormant state where it can avoid immune surveillance. ${ }^{9}$ Despite the clear impact of this infection on public health in endemic regions, we do not understand how $B$. pseudomallei evades the immune response and causes a chronic infection. Chronic melioidosis is frequently misdiagnosed as tuberculosis, and the two diseases share several histological features, ${ }^{2,10}$ suggesting the possibility of common immunological mechanisms between these two infections.

Animal models represent powerful tools to study immunity and virulence factors, and both outbred and inbred strains have been previously described as models

\footnotetext{
Supported by National Institute of Health grant U01Al061363.

Accepted for publication March 29, 2011.

Address reprint requests to Gregory J. Bancroft, Ph.D., Immunology Unit, Department of Infectious \& Tropical Diseases, London School of Hygiene and Tropical Medicine, Keppel St. London WC1E 7HT, United Kingdom. E-mail: gregory.bancroft@Ishtm.ac.uk.
} 
to study human melioidosis. ${ }^{10-15}$ Typically, BALB/c mice, which are very susceptible, have been used to study acute melioidosis. ${ }^{14,16,17}$ In contrast, C57BL/6 mice show increased resistance to $B$. pseudomallei infection but are unable to clear infection, so they are better candidates to mimic chronic infection in humans. ${ }^{11,14}$ The mechanisms that regulate the development of either acute, chronic, or latent melioidosis remain unclear. However, it has been suggested that differential inflammatory responses in BALB/c mice versus C57BL/6 after B. pseudomallei infection may be responsible for different susceptibility to infection, suggesting that high levels of inflammatory cytokines in BALB/c may contribute to the immunopathogenesis. ${ }^{18}$ Other animals such as hamsters, guinea pigs, and rats have also been used, ${ }^{10,19}$ but there is little information on truly chronic models where disease progression is monitored over several months post-challenge. ${ }^{10,20}$

No licensed vaccine exists for either prophylactic or therapeutic use against $B$. pseudomallei. ${ }^{21,22}$ Its high rate of infectivity via the aerosol route and its resistance to many common antibiotics makes this pathogen a potential candidate for bioterrorism. ${ }^{3,22,23}$ Different strategies have been used to develop an effective vaccine, including immunization with heat-killed bacteria, ${ }^{24}$ with live attenuated mutants, ${ }^{25,26}$ or with immunogenic antigens such as lipopolysaccharide (LPS) or outer membrane proteins. ${ }^{27-31}$ Although most have shown some protection, none have reliably achieved sterilizing immunity, instead converting an otherwise overwhelming infection into a chronic disease that is eventually lethal. Thus, persistence in the face of immune or antibiotic pressure is a key feature of melioidosis, but the mechanisms underlying these events are not known.

To study the biology of chronic melioidosis, we infected C57BL/6 mice intranasally with a low dose of $B$. pseudomallei 576 and investigated the histological and inflammatory responses to infection over an extended time course. Our aim was to develop a murine model of chronic disease that reflects chronic disease in humans. In the longer term, this model would be invaluable for evaluating pretreatments and therapeutics that might be used to treat or eliminate chronic $B$. pseudomallei infections.

\section{Materials and Methods}

\section{Bacteria Strain}

B. pseudomallei strain $\mathbf{5 7 6}$ was originally isolated from a patient with a fatal case of human melioidosis in Thailand, and was kindly provided by Dr. Tyrone Pitt (Health Protection Agency, London, UK). Frozen stocks were prepared as previously described. ${ }^{32}$ All procedures involving live bacteria were performed under ACDP (Advisory Committee on Dangerous Pathogens) containment level 3 conditions.

\section{Infection of Mice and Determination of Tissue and Blood Bacterial Load}

Female C57BL/6 mice (6- to 8-week-old; Harlan Laboratories, Bicester, Oxon, UK) were used throughout the studies. All animal experiments were performed in accordance with the guidelines of the Animals (Scientific Procedures) Act of 1986 and were approved by the local ethical review committee at the London School of Hygiene and Tropical Medicine.

A total number of 241 animals were used (191 chronically infected, 10 acutely infected, and 40 uninfected controls), distributed in 11 independent experiments. The number of animals used per experiment and the number of experiments are shown in the figure legends. For survival curves, a total of 76 mice were used. Bacterial load and cytokine production were analyzed at different time points postinfection (p.i.; days 22, 35, 45, and 50) involving 33 and 38 animals, respectively. Some samples used to analyze bacterial burdens were also assayed for cytokines. Histopathology and IHC studies were performed in a total of 79 animals, of which 48 were chronically infected.

For each infection, aliquots were thawed from frozen bacteria stocks and diluted in pyrogen-free saline (PFS). Before intranasal (i.n.) infection, mice were anesthetized intraperitoneally with ketamine $(50 \mathrm{mg} / \mathrm{kg}$; Ketaset; Fort Dodge Animal Health, Southampton, UK) and xylazine (10 mg/kg; Rompun; Bayer, Leverkusen, Germany) diluted in PFS. Challenge was performed by administering i.n. a total volume of $50 \mu \mathrm{L}$ containing 2500 colony-forming units (CFU; acute model) or 100 CFU (chronic model) of $B$. pseudomallei 576. Infection dose was confirmed as described elsewhere. ${ }^{32}$ Control uninfected mice received $50 \mu \mathrm{L}$ of PFS.

At different times p.i., mice were sacrificed, lungs and spleens aseptically removed in cold PBS, and blood collected in $100 \mu \mathrm{L}$ of heparin. Organs were homogenized through a 100- $\mu \mathrm{m}$ cell strainer (BD Biosciences, Oxford, UK), serial 10-fold dilutions of blood and tissue in PBS homogenates were plated onto tryptone soy agar plates (Sigma, Dorset, UK), and bacterial colonies were counted after incubation for 24 to 48 hours at $37^{\circ} \mathrm{C}$. The limits of detection were $50 \mathrm{CFU} /$ organ or $20 \mathrm{CFU} / \mathrm{mL}$ of blood.

\section{Measurement of Spleen and Serum Cytokine and Chemokine Production}

Spleens were harvested in cold PBS containing Complete Mini Protease Inhibitor Cocktail (Roche, Sussex, UK) and homogenized through a cell strainer. Cells were lysed with $0.1 \%$ Triton X-100 (Sigma) before centrifugation at $500 \times g$ for 10 minutes at $4^{\circ} \mathrm{C}$ to pellet cell debris. The homogenates were then assayed for presence of gamma interferon (IFN- $\gamma$ ), IL-6, IL-10, tumor necrosis factor- $\alpha$ (TNF- $\alpha)$, and monocyte chemotactic protein-1 (MCP-1) using Cytometric Bead Array (CBA) Mouse Inflammation Kit (BD Bioscience, Oxford, UK) following the manufacturer's instructions. Sera were collected after 
centrifugation of blood at $500 \times g$ for 10 minutes at $4^{\circ} \mathrm{C}$ and IFN- $\gamma$, IL-6, IL-10, TNF- $\alpha$, and MCP-1 measured using the same CBA Mouse Inflammation Kit as described above.

\section{Histopathological Studies}

Lung, spleen, and liver samples were collected in neutral sodium salt-buffered formalin (Prolabo, Briare, France) at day 3 (p.i.), when animals received an acute challenge (2500 CFU B. pseudomallei) or at days 20 to 60 p.i., when mice were infected with $100 \mathrm{CFU}$ (chronic challenge). Samples were fixed for 1 month, embedded in paraffin wax, and $3-\mu \mathrm{m}$ sections were cut, dewaxed, and rehydrated through xylene and alcohols and then washed in running tapwater for 10 minutes. Hematoxylin and eosin $(\mathrm{H} \& \mathrm{E})$ and Sirius Red histochemical techniques were then performed. Microscopic tissue sections were analyzed by a veterinary pathologist.

\section{Immunohistochemical Studies}

For immunohistochemical detection of B. pseudomallei, a murine monoclonal antibody (3VIE5) that reacts with capsular polysaccharide of $B$. pseudomallei was used (1/2000 dilution). Deparaffinized sections had a purified water rinse, and $20 \mu \mathrm{mol} / \mathrm{L}$ proteinase $\mathrm{K}$ (Novocastra Proteinase K, Newcastle, UK) was applied for 5 minutes at room temperature. The slides were then washed in Tris-buffered saline for 5 minutes and then endogenous peroxidase block was applied for 15 minutes ( $3 \%$ hydrogen peroxide in methanol). Sections were washed in running tapwater for 10 minutes and then clipped into Shandon Sequenza clips (Thermo Fisher Scientific, Loughborough, UK) and washed in Tris-buffered saline for 5 minutes. The Vector M.O.M. Immunodetection kit (Vector, Peterborough, UK) was used. Sections were counterstained with Mayer's hematoxylin and mounted with DPX (BDH, Poole, UK). A mouse IgG2b-negative control (Serotec, Kidlington, UK) was used as the isotype control. Samples were imaged with a Zeiss axioplan 2 microscope (Carl Zeiss Ltd, Welwyn Garden City, UK) fitted with a Retiga 2000R Fast CCD camera (QImaging, Marlow, UK) using Volocity software from Improvision. Images were acquired at $\times 400, \times 200, \times 100$, or $\times 50$.

\section{Statistical Analysis}

Statistical analysis was performed using GraphPad Prism software (GraphPad Software, La Jolla, CA). To compare cytokine and chemokine responses among groups, Kruskal-Wallis test was used followed by Dunn's post test. Data were considered statistically significant when $P<0.05$. The degree of significance was denoted as follows: ${ }^{\star} P<0.05 ;{ }^{\star \star} P<0.01$.

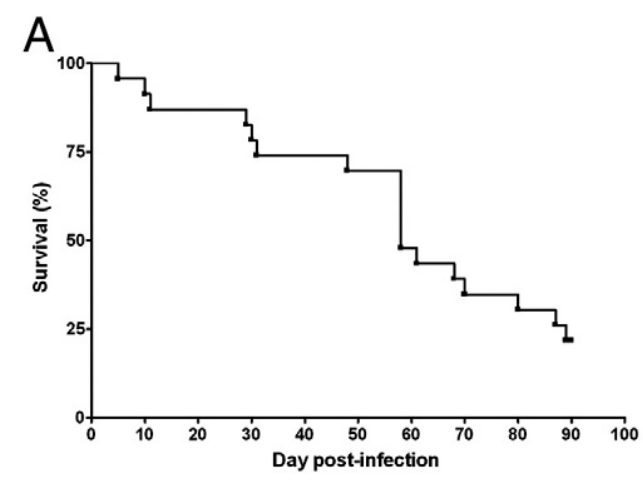

B

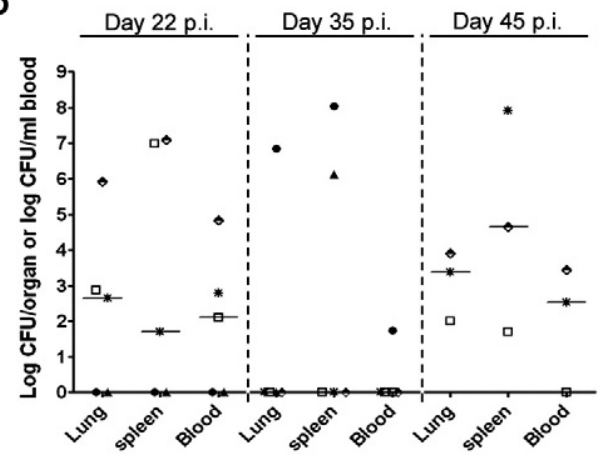

Figure 1. Survival and bacterial burdens after intranasal infection with Burkbolderia pseudomallei. C57BL/6 mice were intranasally infected with 100 colony-forming units (CFU) of B. pseudomallei strain 576. A: Mice were monitored for survival $(n=30)$. B: Bacterial loads in the lung, spleen, and blood at days 22, $35(n=5)$, and $45(n=3)$ postinfection (p.i.). Each symbol represents the number of $\mathrm{CFU}$ of the respective organ from an individual animal. The horizontal line represents the median. Survival data are representative of four independent experiments, and CFU data are representative of three independent experiments.

\section{Results}

\section{Intranasal Challenge with a Low Dose of B. pseudomallei Induces Chronic Infection in Mice That Is Characterized by Differences in Bacterial Load}

To develop a murine model of chronic melioidosis, C57BL/6 mice $(n=30)$ were i.n. challenged with a low dose (100 CFU) of B. pseudomallei 576, and monitored for survival (Figure 1A). A chronic infection was established in which mice started to die from day 10 p.i. onwards, with a median survival time of 58 days. However, $20 \%$ of mice still exhibited no clinical signs of illness 3 months post-challenge when the experiment was terminated. Analysis of lung bacterial loads at day 1 p.i. showed that all animals had detectable bacteria from the initial challenge (data not shown). Analysis of bacterial burdens in lung, spleen, and blood revealed differences in bacterial counts later in the study period between different animals and between organs of the same animal (Figure 1B and Table 1). In many infected animals, bacteria could not be detected after plating tissue samples onto agar from day 20 onwards. It is noteworthy that $35 \%$ of animals with detectable $B$. pseudomallei in lung and/or spleen, were not bacteremic, showing that although the pathogen is 
Table 1. Bacterial Loads and Macroscopic Pathology of Chronically Infected C57BL/6 Mice

\begin{tabular}{|c|c|c|c|c|c|}
\hline Time p.i. (day) & Lung CFU & Blood CFU & Spleen CFU & Splenomegaly & Visible lesion \\
\hline 22 & - & - & - & Yes & No \\
\hline 22 & - & - & - & No & No \\
\hline 22 & $8.5 \times 10^{5}$ & $6.8 \times 10^{4}$ & $1.3 \times 10^{7}$ & Yes & Lung and Spleen \\
\hline 22 & 450 & 610 & 50 & No & No \\
\hline 22 & 750 & 129 & $9.7 \times 10^{6}$ & Yes & Spleen \\
\hline 22 & - & - & $1.2 \times 10^{8}$ & Yes & Spleen \\
\hline 22 & - & - & - & Slightly & No \\
\hline 22 & $1.8 \times 10^{6}$ & 72 & $2.9 \times 10^{8}$ & Yes & Lung and Spleen \\
\hline 22 & - & - & $1.4 \times 10^{8}$ & Yes & Spleen \\
\hline 22 & $3.4 \times 10^{4}$ & 950 & $1.9 \times 10^{5}$ & Yes & Lung and Spleen \\
\hline 35 & - & - & - & No & No \\
\hline 35 & - & - & - & No & No \\
\hline 35 & - & - & $1.4 \times 10^{6}$ & Yes & Spleen \\
\hline 35 & - & - & - & No & No \\
\hline 35 & $7.2 \times 10^{6}$ & 56 & $1.1 \times 10^{8}$ & Yes & Lung and Spleen \\
\hline 45 & 7800 & 2688 & $4.4 \times 10^{4}$ & Yes & Spleen \\
\hline 45 & 100 & - & 50 & No & No \\
\hline 45 & 2350 & 333 & $8.3 \times 10^{7}$ & Yes & Spleen \\
\hline 50 & - & - & $2 \times 10^{8}$ & Yes & Spleen \\
\hline 50 & 8300 & - & $4.7 \times 10^{8}$ & Yes & Spleen \\
\hline 50 & - & 512 & $2.4 \times 10^{8}$ & Yes & Spleen \\
\hline 50 & - & - & $2.8 \times 10^{5}$ & Slightly & Spleen \\
\hline 50 & - & 1632 & $5.4 \times 10^{6}$ & Slightly & Spleen \\
\hline
\end{tabular}

C57BL/6 mice were i.n. infected with 100 CFU B. pseudomallei. At different time points p.i., organs and blood were harvested, bacterial burdens analyzed, and macroscopic lesions recorded.

CFU, colony-forming units; - , not detected.

likely to be spread via the hematogenous route, focal growth of the organism can occur under physical containment. Surprisingly, although mice were challenged via the i.n. route, $41 \%$ of the infected mice in which bacteria could be detected in the spleen did not have culturable organisms in the lung from day 20 onwards.

\section{Chronic Infection Induces the Expression of Proinflammatory Cytokines in Spleens with Lesions and Sera from Bacteremic Mice}

To evaluate cytokine and chemokine responses in chronic infection, serum samples or whole-spleen homogenates were prepared on days 22,35 , or 45 p.i. and assayed ex vivo for IFN- $\gamma$, TNF- $\alpha$, IL-6, MCP-1, and IL-10. Samples were allocated to three groups: uninfected mice, challenged mice without macroscopically visible lesions/splenomegaly or bacteremia, or challenged mice with visible lesions/splenomegaly or bacteremia. Levels of all of the inflammatory cytokines studied (IFN- $\gamma$, TNF- $\alpha$, IL-6, MCP-1) were increased in spleens of challenged mice that had splenomegaly and lesions compared to challenged mice without splenomegaly and lesions, or uninfected mice $(P<0.05$ and $P<0.01$, respectively) (Figure 2, A to D). Increased IFN- $\gamma$ and IL-6 production in the spleen directly correlated with bacterial burden in this organ $(P<0.05 ; r=0.89$ and $r=0.94$, respectively; data not shown). No difference was detected in the production of IL-10 among groups (Figure 2E). Robust proinflammatory cytokine responses were also observed in sera in the presence of bacteremia but not in either uninfected mice or those infected without bacteremia (Figure 3).

\section{Histopathological Analysis of Acute versus Chronic B. pseudomallei-Infected Tissues}

Lung, spleen, and liver are the most common organs affected in human melioidosis. ${ }^{6}$ To analyze the histopathological features of chronic infection, mice were infected intranasally with $100 \mathrm{CFU}$ of B. pseudomallei, organs harvested at day 20 to 60 p.i., and pathology compared to either uninfected mice or mice undergoing an acute septic infection 3 days after a high-dose challenge (2500 CFU).

Previous flow cytometry studies have demonstrated that activated neutrophils are rapidly recruited to the lungs after a high-dose pulmonary challenge with $B$. pseudomallei. ${ }^{15,33}$ Consistent with this, lungs from mice acutely infected showed pulmonary inflammation characterized by multifocal suppurative pneumonia with many neutrophils, both degenerated and viable, and a few lymphocytes and macrophages (Figure 4, B and E). Cell debris was present in the bronchial lumen and perivascular edema and occasional hemorrhages were also observed. In contrast, chronically infected mice developed solid lung lesions that were composed of infiltrating lymphocytes and epithelioid macrophages (Figure 4, C and F). In addition, multinucleated giant cells (MNGC) were detected in some lung lesions (Figure 4F).

We observed four main types of lesions in the lungs of chronically infected mice (Figure 5): Type I, characterized by discrete foci of macrophages and lymphocytes, usually close to blood vessels and the bronchial tree; Type II, round lesions with areas of granulomatous pneumonia with similar distribution to those in Type I, but containing epithelioid macrophages; Type III, large, solid 

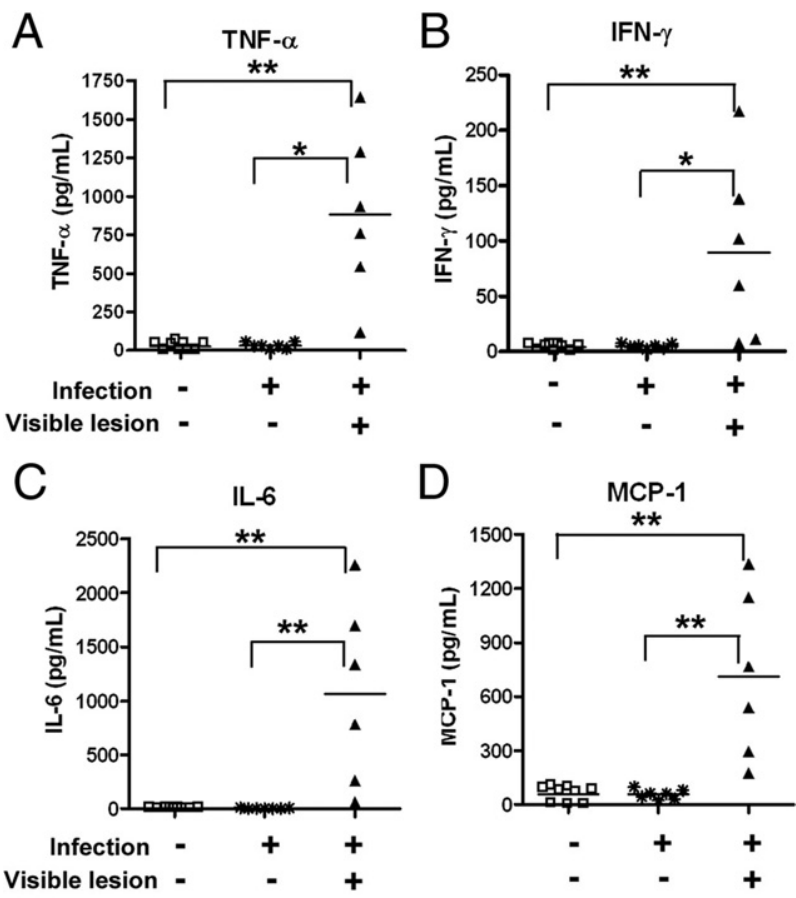

$\mathrm{E}$

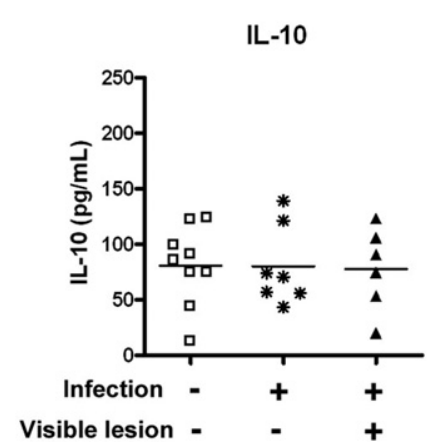

Figure 2. Effect of chronic B. pseudomallei infection on cytokine and chemokine responses in the spleen. At different time points following infection, cytokine protein concentrations from whole-spleen homogenates were measured by cytometric bead array (CBA) analysis. Samples from infected mice were allocated to two groups depending on the presence or absence of macroscopically visible lesions in the organ ( $n=9$ uninfected, $n=13$ infected). A: TNF- $\alpha$. B: IFN- $\gamma$. C: IL-6. D: MCP-1. E: IL-10. Each symbol represents an individual animal. The horizontal line represents the mean. ${ }^{*} P<0.05$, ${ }^{* * *} P<0.01$. Data are representative of two independent experiments.

nonnecrotic granulomas consisting mainly of epithelioid cells and lymphocytes, although a few scattered MNGC were also observed; and Type IV, large granulomas characterized by a caseous necrotic center. Epithelioid macrophages were the main population surrounding the necrotic core in Type IV; however, neutrophils and some lymphocytes and plasma cells were also observed. A distinct fibroblast-rich layer was detected delimiting the necrotic core from nonnecrotic tissue. Thus, both necrotic and solid granulomas develop in the lungs of chronically infected mice although the latter are the most common.

The histology of acutely infected spleens obtained 72 hours p.i. consisted of small inflammatory foci with a central area of necrosis. General lymphoid architecture remained intact, with multifocal necrotic splenitis containing degenerated neutrophils (Figure 4, H and K). By contrast, massive destruction of normal lymphoid structure was observed in the spleens of chronically infected animals (Figure 4, I and L). Splenomegaly and multifocal to coalescent pyogranulomatous splenitis containing a necrotic center were common although nonnecrotic microgranulomas composed of epithelioid macrophages were also observed (data not shown).

The acute inflammatory lesions in the liver were defined by necrotic areas containing degenerated neutrophils and macrophages (Figure 4, N and Q). Hepatic lesions detected in chronically infected mice were mainly large pyogranulomas with a necrotic center that were surrounded by macrophages, lymphocytes, and plasma cells (Figure 4, O and R). However, small pyogranulomas composed mainly of degenerated, but viable, neutrophils, and macrophages were also common. Inflamma-

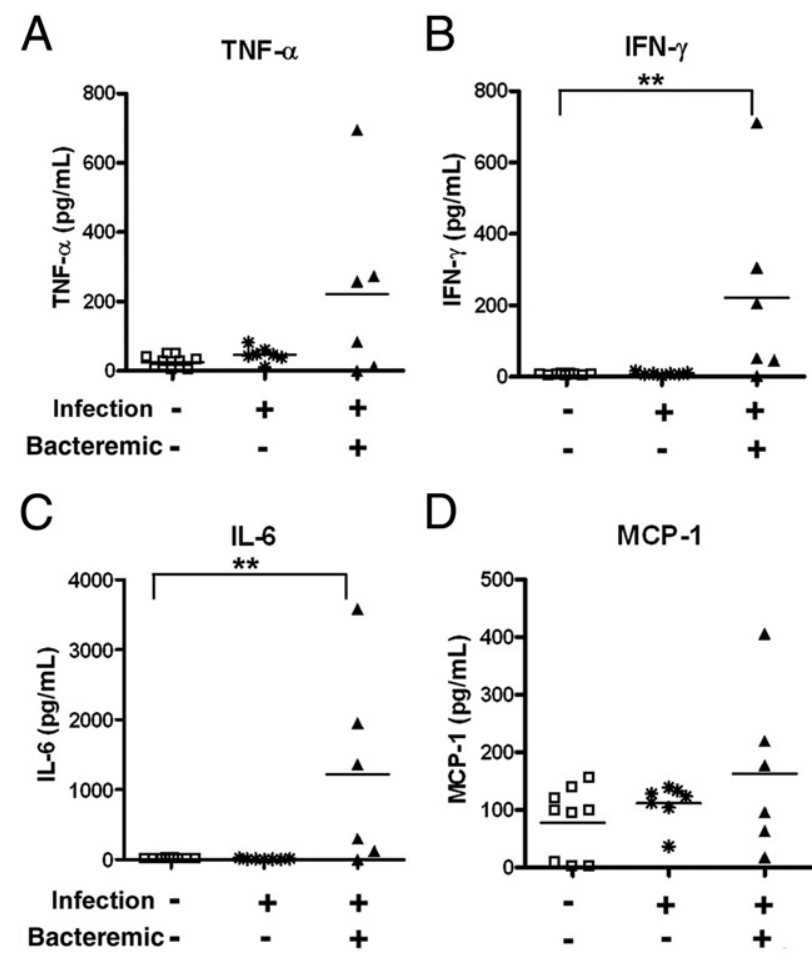

E

\section{IL-10}

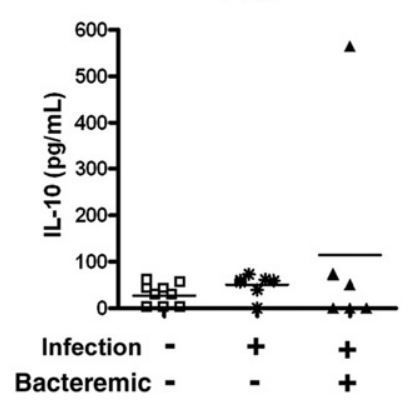

Figure 3. Effect of chronic B. pseudomallei infection on cytokine and chemokine response in the blood. At different time points following infection, cytokine protein concentrations from sera were measured by CBA. Samples ( $n=9$ uninfected, $n=13$ infected) from infected mice were divided into 2 groups according to the presence or absence of bacteria in the blood. A: TNF- $\alpha$. B: IFN- $\gamma$. C: IL-6. D: MCP-1. E: IL-10. Each symbol represents an individual animal. The horizontal line represents the mean. ${ }^{* * *} P<0.01$. Data are representative of two independent experiments. 

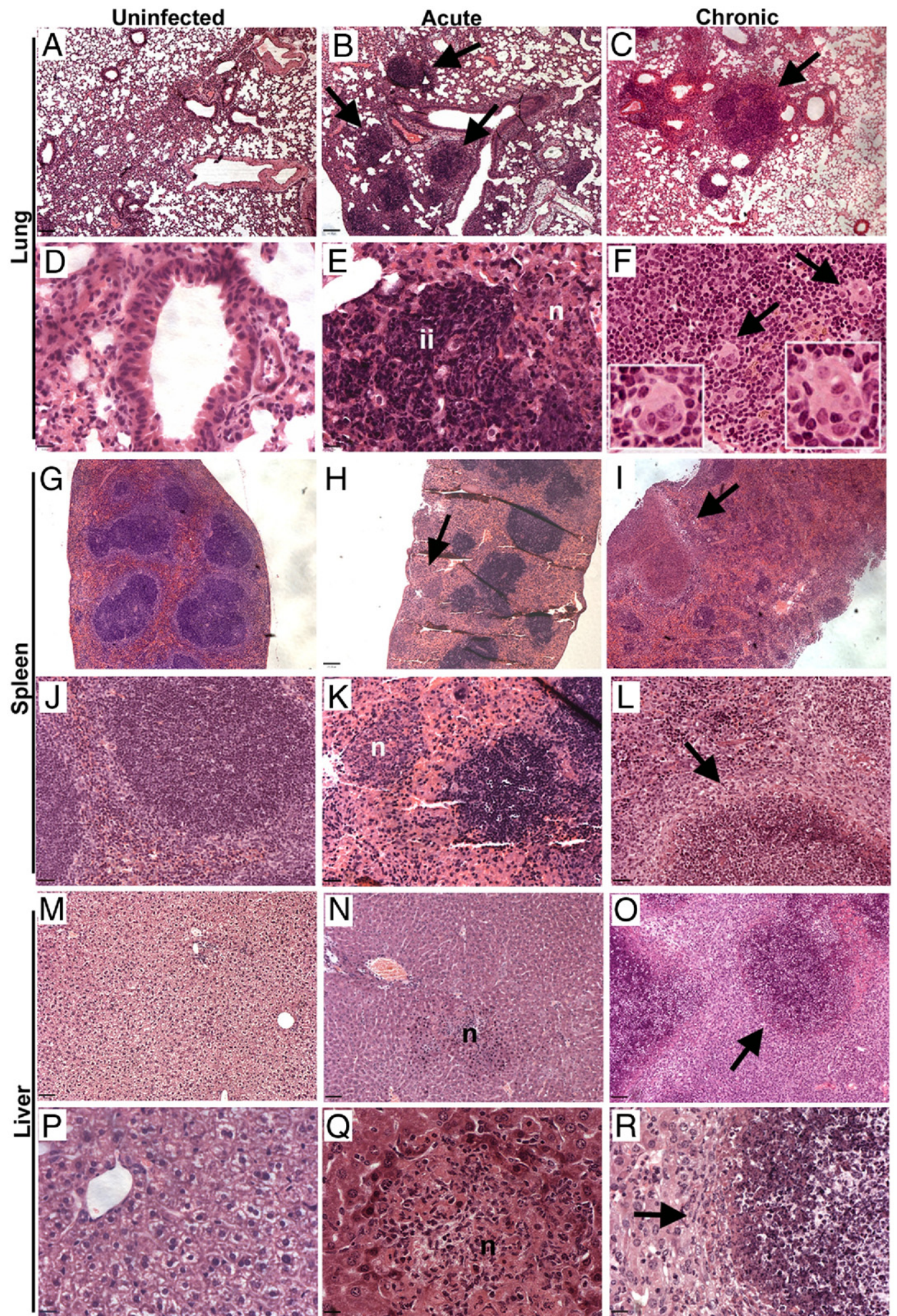

Figure 4. Histopathological changes observed in lung, spleen, and liver of mice infected i.n. with B. psendomallei. C57BL/6 mice were infected with $2500 \mathrm{CFU}$ (acute model, organs collected day $3 \mathrm{pi}, \mathbf{B}, \mathbf{E}, \mathbf{H}, \mathbf{K}, \mathbf{N}$ and $\mathbf{Q}$ ) or $100 \mathrm{CFU}$ (chronic model, organs collected day 20 to $60 \mathrm{pi}, \mathbf{C}, \mathbf{F}, \mathbf{I}, \mathbf{L}, \mathbf{O}$ and $\mathbf{R}$ ). Control mice received PFS (A, D, G, J, M and $\mathbf{P}$ ). B and E: Lungs collected at day 3 p.i. showed multifocal suppurative pneumonia, with areas of inflammatory infiltrates (ii and arrows) which consisted mainly of neutrophils and a few macrophages and lymphocytes, and necrotic areas (n) containing mainly degenerated and viable neutrophils. $\mathbf{C}$ and $\mathbf{F}$ : Lung lesions from chronically infected mice are characterized by nonnecrotic solid lesions, composed of epithelioid macrophages and lymphocytes. F: MNGC are observed in lung lesions (arrows and insets) $\mathbf{H}$ and K: Spleens collected at day 3 p.i. showed multifocal necrotic (n) areas (arrows) with degenerated neutrophils. I and $\mathbf{L}$ : Spleens from chronically infected mice showed multifocal to coalescent pyogranulomatous splenitis. $\mathbf{N}$ and $\mathbf{Q}$ : Livers collected at day 3 p.i. showed multifocal necrotic (n) areas with degenerated neutrophils. $\mathbf{O}$ and $\mathbf{R}$ : Chronic lesions in the liver are characterized by necrotic centers (arrows) containing mainly degenerated but also viable neutrophils; necrotic foci are surrounded by macrophages, lymphocytes, and plasma cells. Sections were stained with H\&E. Scale bars: $140 \mu \mathrm{m}(\mathbf{A}, \mathbf{B}, \mathbf{C}, \mathbf{G}, \mathbf{H}$, and $\mathbf{I}) ; 70 \mu \mathrm{m}(\mathbf{M}, \mathbf{N}$, and $\mathbf{O}) ; 36 \mu \mathrm{m}(\mathbf{J}, \mathbf{K}$, and $\mathbf{L}) ; 18 \mu \mathrm{m}(\mathbf{D}, \mathbf{E}, \mathbf{F}, \mathbf{P}, \mathbf{Q}$ and $\mathbf{R})$. MNGC, multinucleated giant cells; PFS, pyrogen-free saline; p.i., postinfection. Data are representative of three independent experiments $(n=5$ mice, acute model; $n=8$ to 28 mice/experiment, chronic model). 


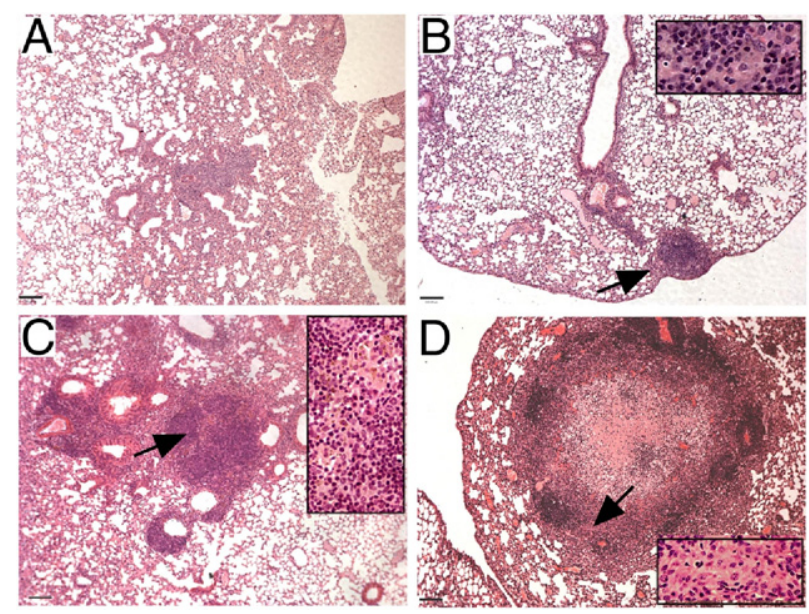

Figure 5. - Representative images of the four types of pulmonary lesions after B. pseudomallei infection. A: Type I is characterized by a discrete focus of macrophages and lymphocytes. B: Type II, area of focal pneumonia composed of numerous lymphocytes and epithelioid macrophages. C: Type III, solid nonnecrotic granuloma consists mainly of epithelioid macrophages and lymphocytes; MNGC are observed in this stage. D: Type IV, large granuloma with necrotic center surrounded by epithelioid macrophages, neutrophils, and some lymphocytes and plasma cells. Arrows point to epithelioid macrophages that are shown at higher magnification in insets. Sections were stained with H\&E. Scale bar $=140 \mu \mathrm{m}$. Data are representative of three independent experiments ( $n=8$ to 28 mice/experiment).

tory infiltrates surrounding the blood vessels in the liver were found in both acute and chronic infection (data not shown). In general, liver chronic lesions were very similar to those present in the spleen.

Although mice were challenged intranasally, only 17 of $45(38 \%)$ chronically infected mice evaluated for histology showed lung lesions, whereas 41 of 44 (93\%) mice had spleen lesions and $100 \%$ had liver lesions. Chronic infection induces splenomegaly, massive destruction of spleen tissue, and high bacterial loads in this organ, suggesting that the spleen, and to a lesser extent the liver, are the most permissive organs for bacterial growth in this chronic model. Furthermore, 17 of 47 (36\%) animals had lost weight at the time of collecting the organs, which is a sign of systemic illness.

\section{Chronic Infection with B. pseudomallei Induces a Fibrotic Response}

The development of a fibrotic capsule containing the foci of bacterial replication is a common feature in other chronic bacterial infections. ${ }^{34-36}$ To see whether $B$. pseudomallei infection also induced this response, lung, liver, and spleen sections from acute or chronically infected mice were stained with Sirius Red to detect the deposition of collagen. Despite extensive tissue pathology and high bacterial burdens, no collagen was detected at day 3 p.i. in any of the organs studied when mice received an acute challenge (data not shown). However, collagen was readily detected in chronically infected mice, forming irregular cords of variable thickness surrounding numerous lesions. This fibrotic capsule was observed in necrotic lung granulomas surrounding the necrotic core but was not present in solid nonnecrotic lesions (Figure 6,
A, D, G, and J). In spleen and liver lesions, a fibrotic structure was also observed surrounding well-defined isolated lesions that contained a necrotic center (Figure $6, \mathrm{~B}, \mathrm{C}, \mathrm{E}$, and F); however, most coalescent and fused pyogranulomas were not encapsulated (Figure $6, \mathrm{H}, \mathrm{I}, \mathrm{K}$, and $\mathrm{L})$.

Most chronic lesions in the spleen and liver were characterized by a necrotic, highly acidophilic center, typically surrounded by a fibrotic structure composed of collagen (Figure 7). Fibroblasts were the main cell population found in this physical barrier that separated the necrotic tissue from nonnecrotic tissue where neutrophils and macrophages were the main population recruited. Epithelioid macrophages were also common within the fibroblast barrier. Finally, lymphocytes and plasma cells were found in abundance in the periphery of the lesions surrounding neutrophils and macrophages.

\section{Location of B. pseudomallei in Chronic Infection}

The number and size of tissue lesions in chronically infected mice were directly related to the bacterial loads in these tissues. To determine the location of B. pseudomallei within these lesions, lung, liver, and spleen sections were initially stained with Gram Twort, but no bacteria were detected (data not shown). However, immunohistochemical staining using the monoclonal antibody 3VIE5, which recognizes an exopolysaccharide antigen from $B$. pseudomallei, revealed large amounts of bacterial antigen in lung, liver, and spleen lesions. This material was located mainly within the cytoplasm of macrophages in lung lesions (Figure 8, D and G). It was also observed within dead and viable macrophages surrounding the areas of necrosis in liver and spleen pyogranulomas, within the fibrotic rim in those lesions that are encapsulated, although it was also observed in some recruited macrophages outside of, but close to, the lesions (Figure 8, E, F, H, and I). No antigen was detected in either uninfected controls, healthy tissue in any of the infected organs, or when compared to sections stained with the isotype control (Figure 8, A, B, and C).

\section{Discussion}

In humans and experimental animals, sterilizing immunity against $B$. pseudomallei is difficult to achieve, and persistence of bacteria either subclinically or as a chronic localized infection is common. However, the mechanisms that underlie persistence and the immune responses that develop during chronic melioidosis are not understood. To address these issues, we have developed a model of chronic experimental melioidosis by infecting C57BL/6 mice via the i.n. route to mimic pulmonary exposure in endemic regions. By combining a low challenge dose and a genetically resistant mouse strain, we were able to generate a chronic infection that persisted for over 3 months.

A key feature of this model was the heterogeneity of disease progression within the infected cohorts. At each 

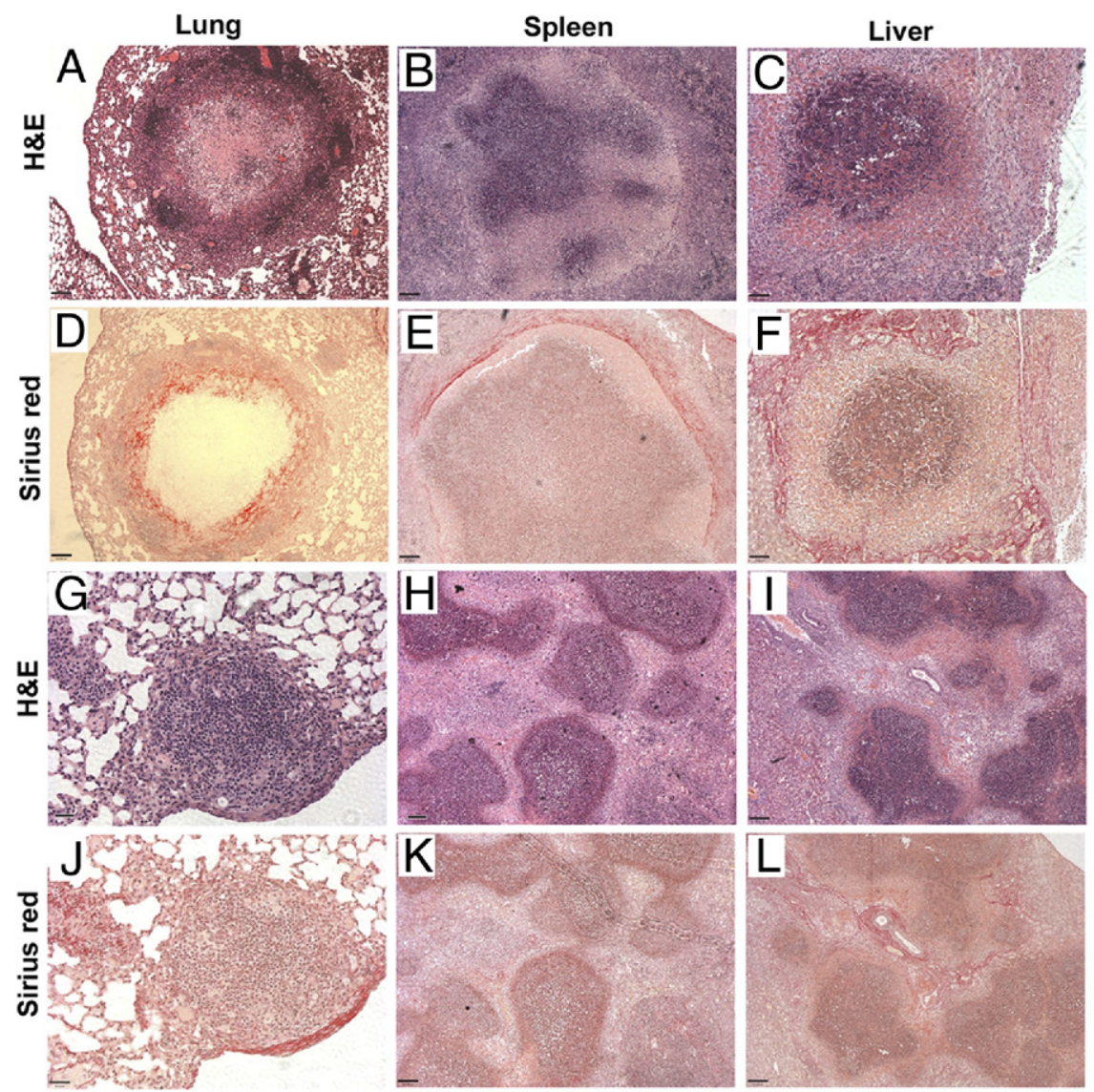

Figure 6. Effect of chronic B. pseudomallei infection on collagen deposition in lung, spleen, and liver. $\mathrm{C} 57 \mathrm{BL} / 6$ mice were infected with 100 CFU of $B$. pseudomallei and organs collected day 20 to 60 p.i. A and D: Lung granulomas containing necrotic center were surrounded by a rim of collagen. $\mathbf{G}$ and $\mathbf{J}$ : Solid nonnecrotic lung lesions were not encapsulated by a fibrotic structure. $\mathbf{B}$ and $\mathbf{E}$ : Numerous spleen and liver $(\mathbf{C}$ and $\mathbf{F})$ necrotic lesions were contained in a collagen matrix. However, most coalescent/ fused lesions, both in the spleen $(\mathbf{H}$ and $\mathbf{K})$ and the liver (I and $\mathbf{L}$ ), were not surrounded by a rim of collagen. Sections were stained with H\&E (A $\mathbf{B}, \mathbf{C}, \mathbf{G}, \mathbf{H}$, and $\mathbf{I})$ or Sirius $\operatorname{Red}(\mathbf{D}, \mathbf{E}, \mathbf{F}, \mathbf{J}, \mathbf{K}$, and L). Scale bars: $140 \mu \mathrm{m}(\mathbf{A}, \mathbf{B}, \mathbf{D}, \mathbf{E}, \mathbf{H}, \mathbf{I}, \mathbf{K}$, and $\mathbf{L}) ; 70 \mu \mathrm{m}(\mathbf{C}$ and $\mathbf{F}) ; 36 \mu \mathrm{m}(\mathbf{G}$ and $\mathbf{J})$. Results shown are representative of three independent experiments ( $n=8$ to $28 \mathrm{mice} /$ experiment). evaluated time point, some animals had progressed to chronic disease, characterized by weight loss and demonstrable bacterial burdens in the blood, liver, lung, or spleen, whereas others showed no signs of illness and no bacteria could be detected in target organs. We believe the latter reflects a latent infection with persistence of low numbers of bacteria that eventually can reactivate. A variable pattern of disease progression has also been observed in immunized mice after challenge with virulent B. pseudomallei. ${ }^{25,26}$ Interestingly, although mice are much more susceptible to infection via the airways than to intraperitoneal challenge, ${ }^{13,37}$ we found the spleen to be a more permissive site for bacterial replication than the lung in this model. The portal of entry and the $B$. pseudomallei strain used are important factors that may influence the cellular response, pathology, and onset of disease in experimental models. ${ }^{13-15,18,33}$ However, chronic infections have also been observed following intraperitoneal or subcutaneous challenge and with other $B$. pseudomallei strains, suggesting that development of chronic infection is an inherent feature of this pathogen
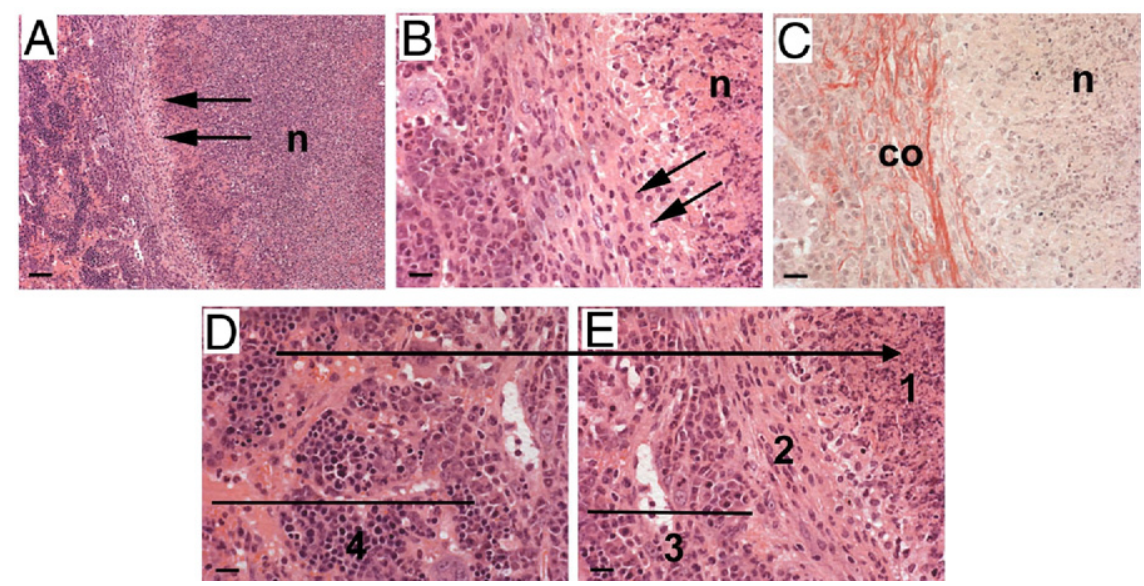

Figure 7. Structure of spleen lesions of mice chronically infected with $B$. pseudomallei. C57BL/ 6 mice were infected with 100 CFU of $B$. pseudomallei and spleens collected day 20 to 60 p.i. A: Spleen section shown represents a granulomatous reaction with necrotic center (n). $\mathbf{B}$ and C: A collagen barrier (co) delimits necrotic and nonnecrotic tissue. $\mathbf{D}$ and $\mathbf{E}$ : Necrotic center (1) is usually encapsulated by a fibrotic capsule (2) that is predominantly surrounded by neutrophils and macrophages (3). Lymphocytes (4) are the main population surrounding the lesions. Epithelioid macrophages are pointed in $\mathbf{A}$ and $\mathbf{B}$ with small arrows. Sections were stained with H\&E (A, B, D, and $\mathbf{E})$ or Sirius Red (C). Scale bars: $70 \mu \mathrm{m}(\mathbf{A}) ; 18 \mu \mathrm{m}(\mathbf{B}, \mathbf{C}, \mathbf{D}$, and $\mathbf{E})$. Co, collagen; $n$, necrotic center. Results are representative of three independent experiments ( $n=8$ to $20 \mathrm{mice} /$ experiment). 


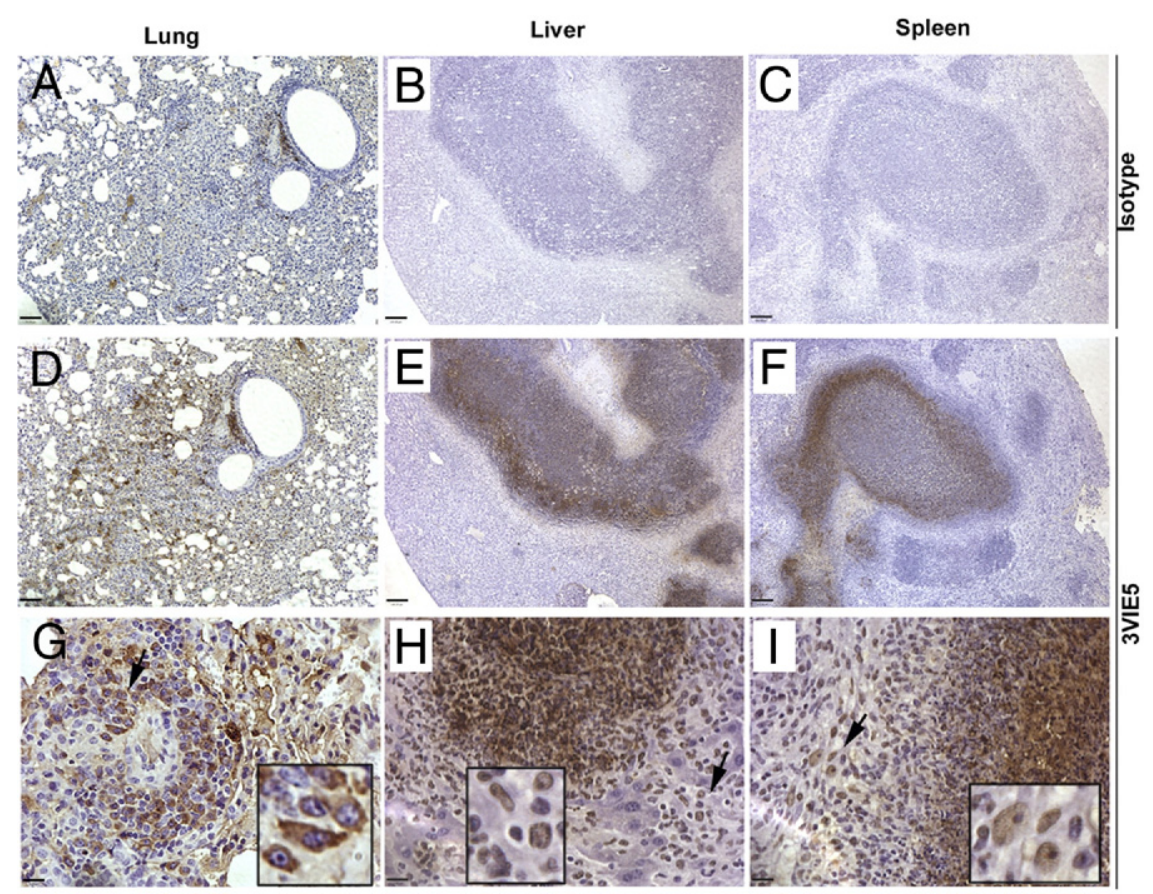

Figure 8. Location of B. pseudomalle $i$ in tissues from chronically infected mice. $\mathrm{C} 57 \mathrm{BL} / 6$ mice were infected with 100 CFU of B. pseudomallei and organs collected at day 20 to 60 p.i. A strong immunoreactivity indicating the presence of the exopolysaccharide antigen from $B$. pseudomallei (brown color) was observed in lung lesions within the cytoplasm of macrophages (D and $\mathbf{G})$, and in liver (E and $\mathbf{H})$ and spleen $(\mathbf{F}$ and $\mathbf{I})$ pyogranulomas, mainly within macrophages (arrows; enlarged in insets) $\mathbf{A}, \mathbf{B}$ and $\mathbf{C}$, isotype control. Scale bars: $140 \mu \mathrm{m}(\mathbf{A}, \mathbf{B}, \mathbf{C}, \mathbf{D}, \mathbf{E}$ and $\mathbf{F}) ; 18 \mu \mathrm{m}(\mathbf{G}, \mathbf{H}$, and $\mathbf{I})$. Data are representative of three different experiments ( $n=8$ to 15 mice/experiment).
(A. Easton, G.J. Bancroft, unpublished data using B. pseudomallei 708A and K96243). ${ }^{10,12}$

Mice that show active disease at the time of harvest (ie, splenomegaly and pyogranulomas and bacteremia) produced proinflammatory cytokines, whereas infected but asymptomatic mice did not. This included IFN- $\gamma$, TNF- $\alpha$, $\mathrm{IL}-6$, and MCP-1, showing similarities to previous reports in acute infections of mice ${ }^{17,18}$ and humans. ${ }^{4,5}$ Previous data from our own and other laboratories have demonstrated the essential role of IFN- $\gamma$ and TNF- $\alpha$ for the control of systemic B. pseudomallei infection, ${ }^{12,32,38}$ suggesting that the production of proinflammatory cytokines is a mechanism induced by the host to try to control the infection. Here, bacterial burdens in the spleen directly correlated with TNF- $\alpha$ levels in the sera $(P<0.05$; data not shown). In addition, IFN- $\gamma$, TNF- $\alpha$, and IL- 6 were increased in sera from mice that were not bacteremic but had spleen lesions, compared to sera from uninfected mice $(P<0.05$; data not shown). This indicates that detection of peripheral blood cytokines can reflect the presence of chronic tissue lesions even in the absence of bacteremia.

Although the cytokine responses of acutely (data not shown) and chronically infected mice were broadly similar, their histopathological features were clearly distinct. In humans, B. pseudomallei causes pyogenic or granulomatous inflammation at virtually any site, but lung, spleen, and liver are the main organs affected. ${ }^{39}$ A combination of both acute necrotizing pneumonia and acute respiratory distress syndrome seem to be responsible for respiratory failure in melioidosis patients. ${ }^{39}$ Here, intranasal challenge of C57BL/6 mice with a high dose of bacteria led to multifocal suppurative pneumonia with many neutrophils but also with macrophages and lymphocytes, similar to that observed in BALB/c mice infected via the aerosol route. ${ }^{13}$ No splenomegaly was observed when mice were acutely infected, and macroscopic lesions could not be detected in either the liver or the spleen. However, microscopic necrotizing multifocal areas containing mainly neutrophils and macrophages were common both in liver and spleen, consistent with previous reports. ${ }^{13,14}$

In contrast, chronic lung pathology was mainly characterized by solid nonnecrotic lesions containing infiltrating lymphocytes and epithelioid macrophages. MNGC, which are present in autopsy samples from humans who have died from melioidosis and in vitro following infection of macrophages with B. pseudomallei, were also observed in some lung lesions. ${ }^{40,41}$ MNGC have not been described in previous animal models of melioidosis, although Dannenberg et al reported the presence of large phagocytic cells containing vacuoles with dark staining phagocytized nuclear debris. ${ }^{42}$ Whether MNGC formation is induced by the pathogen to evade the immune system or whether it is a protective host response remains unclear. ${ }^{43}$ Although not common, we also observed large lung granulomas containing a caseous necrotic center. These resemble the characteristic histological lesions of human tuberculosis and have also been described in human chronic melioidosis. ${ }^{44-46}$ The different types of lung lesions seen in our chronic melioidosis model may reflect progressive stages of granuloma development. Alternatively, as it is proposed for $M$. tuberculosis, initial exposure to the pathogen may induce a primary lesion characterized by an inflammatory focus with central necrosis. Subsequent dissemination from this site in the presence of an ongoing adaptive immune response may then generate secondary lesions composed of macrophages, epithelioid cells, and lymphocytes in the absence of necrosis. ${ }^{47}$

Splenomegaly and massive destruction of lymphoid architecture were also common features of chronically infected animals. ${ }^{42}$ Spleen and liver lesions displayed 
similar patterns, with multifocal to coalescent pyogranulomatous splenitis or hepatitis containing necrotic areas composed of degenerated and viable macrophages and neutrophils. Both macrophages and neutrophils play a role in early resistance against $B$. pseudomallei infection, ${ }^{15,33,48}$ although their role in the control of chronic disease is not known. These cells are also recruited to the spleen and liver in a systemic model of $B$. pseudomallei infection. ${ }^{14}$ Histological analysis of tissue from patients with melioidosis has demonstrated the presence of mainly neutrophils, but also macrophages at infected sites. ${ }^{45,49}$ Nevertheless, our data suggest that despite their recruitment to the site of infection, neutrophils are not sufficient to control the infection.

The presence of epithelioid macrophages, MNGC, and fibrosis in mice chronically infected with $B$. pseudomallei shows parallels to more established models of granuloma formation in other infections such as tuberculosis and schistosomiasis. ${ }^{50,51}$ Here, the organized deposition of collagen that surrounded many of the chronic lesions colocalized with areas of fibroblast recruitment and accurately delimited healthy tissue from areas of necrosis. Not all of the lesions were encapsulated, and these may represent sites of rapid bacterial dissemination that would result in overwhelming infection. Although limiting spread of the bacteria, the fibrotic response may also prevent access of new inflammatory cells, allowing the bacteria to replicate and persist, and potentially impairing the penetration of antibiotics. ${ }^{52}$ Intense immunostaining by a monoclonal antibody specific for the exopolysaccharide antigen of $B$. pseudomallei was observed within chronic necrotic lesions in all tissues, consistent with sustained bacterial growth at these sites. However, when macroscopic lesions were observed, they correlated with a high bacterial load in that organ, suggesting that viable bacteria are confined in granulomas in the lung and pyogranulomas in spleen and liver, and that these lesions provide a suitable niche for the pathogen to persist. $^{53}$

In conclusion, we have developed a murine model of infection with B. pseudomallei that shares features observed in chronic human melioidosis. The histological characteristics of this model are distinct from that observed in acute melioidosis with development of granulomas, caseous necrosis, multinucleated giant cells, and fibrosis that also resemble the pathology of tuberculosis. Van Schaik et al recently reported a novel model of chronic $B$. pseudomallei infection in rats using agar beads that also resulted in granulomatous pathology. ${ }^{10}$ Our murine model provides the additional advantages of allowing use of the wide range of immunological reagents available in this species, does not require the use of agar beads, and allows investigation of chronic infection over more extended periods of time. Testing the paradigms of bacterial persistence and granuloma biology established in tuberculosis for their validity in these models should provide important new insights into the biology of melioidosis.

\section{Acknowledgments}

We thank Robert Gilbert and all of the members of the London School of Hygiene and Tropical Medicine Biological Services Facility for animal husbandry and Dr. Tyrone Pitt (Health Protection Agency, London, UK) for the provision of Burkholderia pseudomallei 576.

\section{References}

1. Wiersinga WJ, van der Poll T: Immunity to Burkholderia pseudomallei Curr Opin Infect Dis 2009, 22:102-108

2. Vidyalakshmi K, Chakrapani M, Shrikala B, Damodar S, Lipika S, Vishal S: Tuberculosis mimicked by melioidosis. Int J Tuberc Lung Dis 2008, 12:1209-1215

3. Wiersinga WJ, van der Poll T, White NJ, Day NP, Peacock SJ: Melioidosis: insights into the pathogenicity of Burkholderia pseudomallei. Nat Rev Microbiol 2006, 4:272-282

4. Friedland JS, Suputtamongkol Y, Remick DG, Chaowagul W, Strieter RM, Kunkel SL, White NJ, Griffin GE: Prolonged elevation of interleukin-8 and interleukin-6 concentrations in plasma and of leukocyte interleukin-8 mRNA levels during septicemic and localized Pseudomonas pseudomallei infection. Infect Immun 1992, 60:2402-2408

5. Lauw FN, Simpson AJ, Prins JM, Smith MD, Kurimoto M, van Deventer SJ, Speelman P, Chaowagul W, White NJ, van der Poll T: Elevated plasma concentrations of interferon (IFN)-gamma and the IFN-gamma-inducing cytokines interleukin (IL)-18. IL-12, and IL-15 in severe melioidosis. J Infect Dis 1999, 180:1878-1885

6. White NJ: Melioidosis. Lancet 2003, 361:1715-1722

7. Maharjan B, Chantratita N, Vesaratchavest M, Cheng A, Wuthiekanun V, Chierakul W, Chaowagul W, Day NP, Peacock SJ: Recurrent melioidosis in patients in northeast Thailand is frequently due to reinfection rather than relapse. J Clin Microbiol 2005, 43:6032-6034

8. Mays EE, Ricketts EA: Melioidosis: recrudescence associated with bronchogenic carcinoma twenty-six years following initial geographic exposure. Chest 1975, 68:261-263

9. Adler NR, Govan B, Cullinane M, Harper M, Adler B, Boyce JD: The molecular and cellular basis of pathogenesis in melioidosis: how does Burkholderia pseudomallei cause disease? FEMS Microbiol Rev 2009, 33:1079-1099

10. van Schaik E, Tom M, DeVinney R, Woods DE: Development of novel animal infection models for the study of acute and chronic Burkholderia pseudomallei pulmonary infections. Microbes Infect 2008, 10: 1291-1299

11. Leakey AK, Ulett GC, Hirst RG: BALB/C and C57BI/6 mice infected with virulent Burkholderia pseudomallei provide contrasting animal models for the acute and chronic forms of human melioidosis. Microb Pathog 1998, 24:269-275

12. Santanirand P, Harley VS, Dance DA, Drasar BS, Bancroft GJ: Obligatory role of gamma interferon for host survival in a murine model of infection with Burkholderia pseudomallei. Infect Immun 1999, 67: 3593-3600

13. Lever MS, Nelson M, Stagg AJ, Beedham RJ, Simpson AJ: Experimental acute respiratory Burkholderia pseudomallei infection in BALB/c mice. Int J Exp Pathol 2009, 90:16-25

14. Barnes JL, Ulett GC, Ketheesan N, Clair T, Summers PM, Hirst RG: Induction of multiple chemokine and colony-stimulating factor genes in experimental Burkholderia pseudomallei infection. Immunol Cell Biol 2001, 79:490-501

15. Easton A, Haque A, Chu K, Lukaszewski R, Bancroft GJ: A critical role for neutrophils in resistance to experimental infection with Burkholderia pseudomallei. J Infect Dis 2007, 195:99-107

16. Hoppe I, Brenneke B, Rohde M, Kreft A, Haussler S, Reganzerowski A, Steinmetz I: Characterization of a murine model of melioidosis: comparison of different strains of mice. Infect Immun 1999, 67:28912900

17. Liu B, Koo GC, Yap EH, Chua KL, Gan YH: Model of differential susceptibility to mucosal Burkholderia pseudomallei infection. Infect Immun 2002, 70:504-511

18. Tan GY, Liu Y, Sivalingam SP, Sim SH, Wang D, Paucod JC, Gauthier $Y$, Ooi EE: Burkholderia pseudomallei aerosol infection results in 
differential inflammatory responses in BALB/C and C57BI/6 mice. J Med Microbiol 2008, 57:508-515

19. Tuanyok A, Tom M, Dunbar J, Woods DE: Genome-wide expression analysis of Burkholderia pseudomallei infection in a hamster model of acute melioidosis. Infect Immun 2006, 74:5465-5476

20. Najdenski H, Kussovski V, Vesselinova A: Experimental Burkholderia pseudomallei infection of pigs. J Vet Med B Infect Dis Vet Public Health 2004, 51:225-230

21. Estes DM, Dow SW, Schweizer HP, Torres AG: Present and future therapeutic strategies for melioidosis and glanders. Expert Rev Anti Infect Ther 2010, 8:325-338

22. Bondi SK, Goldberg JB: Strategies toward vaccines against Burkholderia mallei and Burkholderia pseudomallei. Expert Rev Vaccines 2008, 7:1357-1365

23. Stone R: Infectious disease. Racing to defuse a bacterial time bomb. Science 2007, 317:1022-1024

24. Sarkar-Tyson M, Smither SJ, Harding SV, Atkins TP, Titball RW: Protective efficacy of heat-inactivated B. thailandensis. B mallei or B pseudomallei against experimental melioidosis and glanders. Vaccine 2009, 27:4447-4451

25. Haque A, Chu K, Easton A, Stevens MP, Galyov EE, Atkins T, Titball R, Bancroft GJ: A live experimental vaccine against Burkholderia pseudomallei elicits CD4+ T cell-mediated immunity, priming T cells specific for 2 type III secretion system proteins. J Infect Dis 2006, 194:1241-1248

26. Breitbach K, Kohler J, Steinmetz I: Induction of protective immunity against Burkholderia pseudomallei using attenuated mutants with defects in the intracellular life cycle. Trans R Soc Trop Med Hyg 2008 102 Suppl 1:S89-S94

27. Nelson M, Prior JL, Lever MS, Jones HE, Atkins TP, Titball RW: Evaluation of lipopolysaccharide and capsular polysaccharide as subunit vaccines against experimental melioidosis. J Med Microbio 2004, 53:1177-1182

28. Hara Y, Mohamed R, Nathan S: Immunogenic Burkholderia pseudomallei outer membrane proteins as potential candidate vaccine targets. PLoS One 2009, 4:e6496

29. Harland DN, Chu K, Haque A, Nelson M, Walker NJ, Sarkar-Tyson M, Atkins TP, Moore B, Brown KA, Bancroft G, Titball RW, Atkins HS: Identification of a LolC homologue in Burkholderia pseudomallei, a novel protective antigen for melioidosis. Infect Immun 2007, 75:41734180

30. Rozak DA, Gelhaus HC, Smith M, Zadeh M, Huzella L, Waag D, Adamovicz JJ: CpG oligodeoxyribonucleotides protect mice from Burkholderia pseudomallei but not Francisella tularensis Schu S4 aerosols. J Immune Based Ther Vaccines 2010, 8:2

31. Elvin SJ, Healey GD, Westwood A, Knight SC, Eyles JE, Williamson ED: Protection against heterologous Burkholderia pseudomallei strains by dendritic cell immunization. Infect Immun 2006, 74:17061711

32. Haque A, Easton A, Smith D, O'Garra A, Van Rooijen N, Lertmemongkolchai G, Titball RW, Bancroft GJ: Role of T cells in innate and adaptive immunity against murine Burkholderia pseudomallei infection. J Infect Dis 2006, 193:370-379

33. Wiersinga WJ, Wieland CW, Roelofs JJ, van der Poll T: MyD88 dependent signaling contributes to protective host defense against Burkholderia pseudomallei. PLoS One 2008, 3:e3494

34. Dheda K, Booth H, Huggett JF, Johnson MA, Zumla A, Rook GA: Lung remodeling in pulmonary tuberculosis. J Infect Dis 2005, 192: 1201-1209
35. Ellis JA: Immunophenotype of pulmonary cellular infiltrates in sheep with visceral caseous Iymphadenitis. Vet Pathol 1988, 25:362-368

36. Dagleish MP, Finlayson J, Bayne C, MacDonald S, Sales J, Hodgson $\mathrm{JC}$ : Characterization and time course of pulmonary lesions in calves after intratracheal infection with Pasteurella multocida A:3. J Comp Pathol 2010, 142:157-169

37. Titball RW, Russell P, Cuccui J, Easton A, Haque A, Atkins T, SarkarTyson M, Harley V, Wren B, Bancroft GJ: Burkholderia pseudomallei: animal models of infection. Trans R Soc Trop Med Hyg 2008, 102 Suppl 1:S111-S116

38. Barnes JL, Williams NL, Ketheesan N: Susceptibility to Burkholderia pseudomallei is associated with host immune responses involving tumor necrosis factor receptor-1 (TNFR1) and TNF receptor-2 (TNFR2). FEMS Immunol Med Microbiol 2008, 52:379-388

39. Dance DA: Melioidosis. Curr Opin Infect Dis 2002, 15:127-132

40. Harley VS, Dance DA, Drasar BS, Tovey G: Effects of Burkholderia pseudomallei and other Burkholderia species on eukaryotic cells in tissue culture. Microbios 1998, 96:71-93

41. Kespichayawattana W, Rattanachetkul S, Wanun T, Utaisincharoen $P$, Sirisinha S: Burkholderia pseudomallei induces cell fusion and actinassociated membrane protrusion: a possible mechanism for cell-tocell spreading. Infect Immun 2000, 68:5377-5384

42. Dannenberg AM Jr., Scott EM: Melioidosis: pathogenesis and immunity in mice and hamsters. I Studies with virulent strains of Malleomyces pseudomallei. J Exp Med 1958, 107:153-166

43. Varin A, Gordon S: Alternative activation of macrophages: immune function and cellular biology. Immunobiology 2009, 214:630-641

44. Saunders BM, Britton WJ: Life and death in the granuloma: immunopathology of tuberculosis. Immunol Cell Biol 2007, 85:103-111

45. Piggott JA, Hochholzer L: Human melioidosis. A histopathologic study of acute and chronic melioidosis. Arch Pathol 1970, 90:101111

46. Barry CE 3rd, Boshoff HI, Dartois V, Dick T, Ehrt S, Flynn J, Schnappinger D, Wilkinson RJ, Young D: The spectrum of latent tuberculosis: rethinking the biology and intervention strategies. Nat Rev Microbiol 2009, 7:845-855

47. McMurray DN: Hematogenous reseeding of the lung in low-dose, aerosol-infected guinea pigs: unique features of the host-pathogen interface in secondary tubercles. Tuberculosis (Edinb) 2003, 83:131134

48. Breitbach K, Klocke S, Tschernig T, van Rooijen N, Baumann U, Steinmetz I: Role of inducible nitric oxide synthase and NADPH oxidase in early control of Burkholderia pseudomallei infection in mice. Infect Immun 2006, 74:6300-6309

49. Wong KT, Puthucheary SD, Vadivelu J: The histopathology of human melioidosis. Histopathology 1995, 26:51-55

50. Cosma CL, Sherman DR, Ramakrishnan L: The secret lives of the pathogenic mycobacteria. Annu Rev Microbiol 2003, 57:641-676

51. Wilson MS, Mentink-Kane MM, Pesce JT, Ramalingam TR, Thompson R, Wynn TA: Immunopathology of schistosomiasis. Immunol Cell Biol 2007, 85:148-154

52. Davis JM, Ramakrishnan L: The role of the granuloma in expansion and dissemination of early tuberculous infection. Cell 2009, 136: 37-49

53. Lever MS, Nelson M, Ireland PI, Stagg AJ, Beedham RJ, Hall GA, Knight G, Titball RW: Experimental aerogenic Burkholderia mallei (glanders) infection in the BALB/C mouse. J Med Microbiol 2003, 52:1109-1115 